\title{
Effet anti-inflammatoire et cicatrisant des extraits aqueux et éthanolique des écorces du tronc de Buchholzia coriacea Engl. (Capparidaceae)
}

\author{
Epa Charles ${ }^{1}$, Elion Itou RDG ${ }^{2}$, Etou Ossibi AW², Attibayéba ${ }^{3 *}$, Ongoka Pascal Robin ${ }^{1}$, Abena Ange \\ Antoine ${ }^{2}$ \\ 1'Département des Sciences Naturelles, École Normale Supérieure, Université Marien Ngouabi Congo, B.P 69, \\ Brazzaville - Congo. \\ 2Laboratoire de Biochimie et Pharmacologie, Faculté des Sciences de la Santé, Université Marien Ngouabi Congo, \\ B.P 69, Brazzaville - Congo. \\ ${ }^{3 *}$ Laboratoire de Physiologie et Production Végétales, Faculté des Sciences et Techniques, Université Marien \\ Ngouabi, B.P 69, Brazzaville - Congo. \\ *Auteur correspondant : Attibayéba : e-mail : attisattibayeba@yahoo.com ou attibayeba@gmail.com
}

Original submitted in on $27^{\text {th }}$ August 2015. Published online at www.m.elewa.org on $31^{\text {st }}$ October 2015

http://dx.doi.org/10.4314/jab.v94i1.9

\begin{abstract}
RESUME
Objectif : La présente étude a pour objectif d'étudier les effets anti-inflammatoire et cicatrisant des écorces du tronc de Buchholzia coriacea Engl. (Capparidaceae) appelé Ombanda en langue Téké afin de valoriser les plantes médicinales de la flore spontanée congolaise.

Méthodologie et résultats : La méthodologie consiste à étudier l'effet anti-inflammatoire en mesurant le volume de l'œdème de la patte ayant reçu la carragénine $1 \%$ à l'aide d'un Pléthismomètre de type Ugo Basile 7140. Par ailleurs, des plaies d'incision circulaire de $2 \mathrm{~cm}$ de diamètre étaient faites chez les rats Wistar pour évaluer l'activité cicatrisante de l'extrait éthanolique pâteux des écorces de $B$. coriacea à $200 \mathrm{mg} / \mathrm{kg}$. Les résultats montrent que les extraits aqueux et éthanolique des écorces du tronc de B. coriacea aux doses de 200 et 400 $\mathrm{mg} / \mathrm{kg}$ s'opposent à l'augmentation du volume de l'œdème de la patte du rat induit par la carragénine $1 \%$. Le pourcentage d'inhibition du volume de l'œdème avec l'extrait éthanolique (200 mg/kg) est de 87,34 \% à la 6 ème heure alors qu'avec l'extrait aqueux à la même heure et avec la même faible dose il est de 72,56 \%. Concernant l'activité cicatrisante, les résultats montrent que l'application de l'extrait éthanolique pâteux des écorces de B.coriacea à $200 \mathrm{mg} / \mathrm{kg}$ sur les plaies provoque leur cicatrisation au bout de 18 jours.

Conclusion et application : Les résultats de l'étude montrent que les extraits aqueux et éthanolique des écorces du tronc de Buchholzia coriacea Engl. (Capparidaceae) ont des propriétés cicatrisantes et anti-inflammatoires qui pourraient justifier l'utilisation de cette plante en médecine traditionnelle contre les maladies inflammatoires. Mots clés : Buchholzia coriacea Engl., écorces du tronc, inflammation, cicatrisation, rats Wistar
\end{abstract}


Anti-inflammatory and healing effects of aqueous and ethanolic extracts of stem bark of Buchholzia coriacea Engl. (Capparidaceae)

\section{ABSTRACT}

Objective: The aims of this study is to evaluate anti-inflammatory and healing effects of stem bark of Buchholzia coriacea Engl.(Capparidaceae) invited Ombanda in Teke language in order to develop the medicinal plants of Congolese flora.

Methodology and results: Inflammatory was induced by administration sub-plantar of $0.05 \mathrm{ml}$ of $1 \%$ carrageenan at the right paw. Oedema was measured using an Ugo Basile 7140 pletysmometer, Italy. In addition, wounds circular incision of $2 \mathrm{~cm}$ in diameter were made in the Wistar rats to evaluate the healing activity of ethanolic extract of the stem barks of Buchholzia coriacea at dose of $200 \mathrm{mg} / \mathrm{kg}$. The results show that the aqueous and ethanolic extracts of stem barks of B coriacea at dose of 200 and $400 \mathrm{mg} / \mathrm{kg}$ are opposed to the increase oedema induced by the carrageenan $1 \%$ in rats. The percentage of inhibition of the oedema volume with ethanolic and aqueous extract at the dose of $200 \mathrm{mg} / \mathrm{kg}$ per sixth hour is respectively 87.34 and $72.56 \%$. Concerning the healing activity, the results show that the application of the ethanolic extract of stem barks of B. coriacea at dose of $200 \mathrm{mg} / \mathrm{kg}$ on the wounds causes their cicatrization at the end of 18 days.

Conclusion and application: Aqueous and ethanolic extracts of stem barks of Buchholzia coriacea Engl (Capparidaceae) show healing properties and anti-inflammatory effect. These results could justify the use of this plant in traditional medicine against the inflammatory diseases.

Key words: Buchholzia coriacea, cicatrization, inflammation, rats stem barks.

\section{INTRODUCTION}

L'organisation mondiale de la santé (OMS) éprouve aujourd'hui d'énormes difficultés à couvrir la santé pour tous, en raison de la persistance et la recrudescence des maladies chroniques et à cause de l'apparition de nombreuses maladies non maîtrisées (OMS, 2013). Parmi ces maladies chroniques, on peut citer les maladies inflammatoires. Les regards de nombreuses populations d'Afrique, estimées à environ $80 \%$, sont de plus en plus tournés vers la médecine traditionnelle qui utilise les plantes médicinales pour soulager ou guérir beaucoup de pathologies. Au nombre de ces plantes, existe Buchholzia coriacea Engl. (Capparidaceae). Arbre à feuillage persistant pouvant atteindre $25 \mathrm{~m}$ de haut, Buchholzia coriacea pousse dans les forêts tropicales (Figure 1). II est rencontré en Guinée, au Gabon, au Cameroun, au Ghana, au Libéria; les graines de cette plante (Figure 2) sont utilisées en médecine traditionnelle africaine contre de nombreuses pathologies (Ezekiel et al, 2009 ; Ezeja et al, 2011 ; Enechiand Nwodo (2014) ; Nwachukwu et al, 2014). En République du Congo, les écorces du tronc de $B$. coriacea sont habituellement utilisées contre les maladies inflammatoires, les douleurs, les infections microbiennes et la fièvre (Bouquet et Jacquot, 1967 ; Diafouka, 1997 ; Bouquet, 1969 ; Adjanohoun et al, 1988). La présente étude vise à étudier les effets anti-inflammatoire et cicatrisant des écorces du tronc de B. coriacea. 


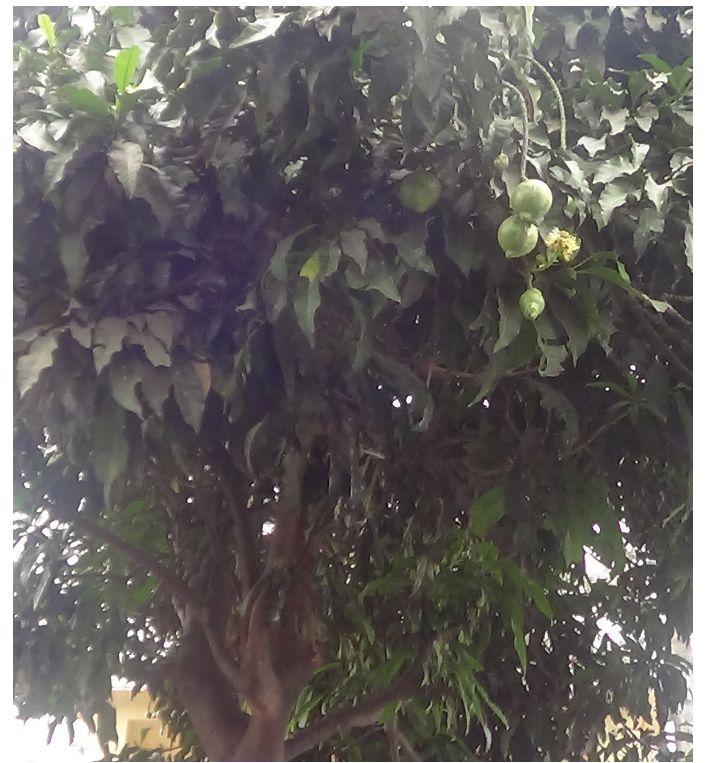

Figure 1: Arbre de Buchholzia coriacea Engl. (Capparidaceae)

\section{MATERIEL ET METHODES \\ Matériel}

Matériel végétal : Les écorces du tronc de $B$. coriacea ont été utilisées. Ces écorces ont été récoltées à Brazzaville au mois d'août 2014. Les échantillons récoltés, ont été identifiés et comparés à l'échantillon de référence $\mathrm{N}^{\circ} 2456$ de la date du 17-2-1968 de l'herbarium du Centre d'Étude sur les Ressources Végétales (C.E.R.VE) de Brazzaville (CONGO). Ensuite, ils ont été séchés au laboratoire des Sciences de la Vie et de la Terre de l'École Normale Supérieure (Université Marien Ngouabi, Brazzaville - Congo) à l'abri du soleil, à la température ambiante.

Matériel animal : Les souris albinos Swiss (mâles et femelles) de poids variant entre 20 et $30 \mathrm{~g}$ et les rats Wistar randomisés (mâles et femelles) de poids compris entre 180 et $210 \mathrm{~g}$ ont été utilisées. Ces animaux ont été élevés à l'animalerie de la Faculté des Sciences de la Santé (Université Marien Ngouabi, Brazzaville - Congo), dans des conditions standard d'éclairement (12 heures d'éclairement blanc, 12 heures d'obscurité) et à la température ambiante $\left(25 \pm 1^{\circ}\right)$. Ils recevaient une nourriture standard et s'abreuvaient à volonté avec l'eau du robinet.

\section{Méthodes}

\section{Préparation des extraits}

Extrait aqueux: Un aliquot de $100 \mathrm{~g}$ de poudre d'écorces du tronc de $B$. coriacea ont été homogénéisés pendant 24 heures dans $1000 \mathrm{ml}$ d'eau distillée. Le

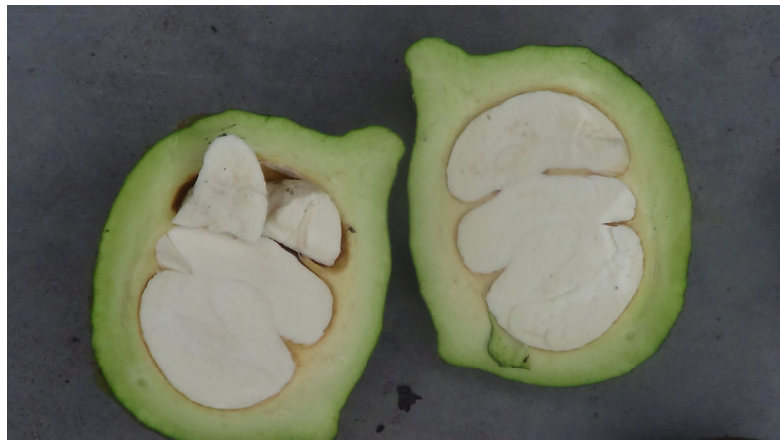

Figure 2 : graines du fruit de Buchholzia coriacea Engl. (Capparidaceae)

mélange obtenu a été porté à ébullition dans un chauffeballon pendant 30 minutes. Après refroidissement et filtration sur papier wattman, le décocté a été évaporé à l'étuve à la température de $70^{\circ}$. Le décocté concentré, en poudre, obtenu était conservé dans un flacon sec et stérile au réfrigérateur pour les tests pharmacologiques. Extrait éthanolique : L'extrait éthanolique a été préparé par macération. Ainsi, $500 \mathrm{~g}$ d'écorces du tronc de $B$. coriacea pulvérisés ont été homogénéisés dans $5000 \mathrm{ml}$ d'éthanol à $96^{\circ}$ pendant $72 \mathrm{~h}$ sous agitation magnétique, puis filtrés sur le coton hydrophile et du papier Wattman. Le filtrat obtenu a été évaporé à $40^{\circ} \mathrm{C}$ à l'aide d'un rotavapor Buchi R-10 de marque Suisse. Le macéré concentré, en poudre, obtenu était conservé dans un flacon sec et stérile au réfrigérateur pour les tests pharmacologiques.

Effet des extraits aqueux et éthanolique des écorces du tronc de B.coriacea sur l'inflammation aiguë induite par la carragénine $1 \%$ chez le rat : La méthode décrite par (Elion Itou et al, 2014) a été utilisée. Ainsi, 35 rats Wistar mis à jeûn 24 heures avant l'expérimentation ont été répartis en 7 lots de 5 rats chacun. Les lots 1 et 2 témoins avaient reçu respectivement par voie orale (p.o) l'eau distillée (témoin de l'extrait aqueux) et la solution de $\mathrm{NaCl}$ à $0,9 \%$ (témoin de l'extrait éthanolique) aux doses de $0,5 \mathrm{ml} / 100 \mathrm{~g}$ et $1 \mathrm{ml} / 100 \mathrm{~g}$. Le lot 3 a reçu le Diclofénac (molécule de référence) à $5 \mathrm{mg} / \mathrm{kg}$, p.o. Les lots 4,5 et 6 , 7 avaient reçu respectivement l'extrait aqueux et l'extrait 
éthanolique des écorces de tronc de $B$. coriacea aux doses de 200 et $400 \mathrm{mg} / \mathrm{kg}$, p.o. Une heure après les différents traitements, la carragénine à $1 \%$ leur a été injectée sous le coussinet plantaire à la dose de $0,05 \mathrm{ml}$. L'effet anti-inflammatoire a été évalué en mesurant le volume de l'œdème de la patte ayant reçu la carragénine $1 \%$ à 30 min. $1 \mathrm{~h}, 2 \mathrm{~h}, 3 \mathrm{~h}, 4 \mathrm{~h}, 5 \mathrm{~h}$ et $6 \mathrm{~h}$ à l'aide d'un Pléthismomètre de type Ugo Basile 7140 (Elion Itou et al, 2014). La formule courante de calcul du pourcentage d'inhibition (\% I) a été appliquée (Elion Itou et al, 2014). Étude des effets curatifs de l'extrait éthanolique pâteux sur les plaies induites chez les rats wistar: Cette étude a été réalisée avec l'extrait éthanolique pâteux (selon sa consistance) de $200 \mathrm{mg} / \mathrm{kg}$, préparé dans les conditions précédemment mentionnées. Le protocole décrit par Sagliyan et al, (2010) a été utilisé. Une plaie d'incision circulaire de $2 \mathrm{~cm}$ de diamètre était faite dans la région dorso-omoplate de chaque de rat anesthésié à l'éther. Trois lots de 5 rats chacun placés dans des cages individuelles ont été respectivement traités, par application topique directe, avec la solution de $\mathrm{NaCl} 0,9 \%$ à $10 \mathrm{ml} / \mathrm{kg}$, de $2 \mathrm{ml}$ de la solution de bétadine de commerce et l'extrait éthanolique pâteux des écorces de $B$. coriacea à $200 \mathrm{mg} / \mathrm{kg}$. Le traitement et les mesures du diamètre des plaies ont été faits tous les deux jours pendant 28 jours à l'aide d'une règle graduée. Tous les animaux ont été suivis régulièrement jusqu'à la cicatrisation complète des plaies et ont eu accès à la nourriture et à l'eau à volonté. Les plaies des animaux étaient désinfectées tous les jours durant la période de l'expérimentation avec de l'alcool à $95^{\circ}$. Le pourcentage de rétrécissement ou de contraction des plaies a été calculé selon la formule suivante :

\section{Poucentage de la contraction $=\frac{\text { Surfare de la plaie rinatrigée }}{\text { surface de la plaie initiale }} \times 100$}

Analyse statistique des résultats : Les résultats sont exprimés en moyennes plus ou moins erreurs standards

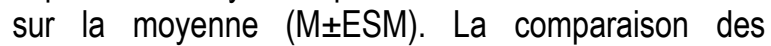
moyennes entre les lots traités et témoins, a été faite par

\section{RESULTATS}

Effet de l'extrait aqueux des écorces du tronc de $B$. coriacea sur l'inflammation aiguë induite par la carragénine $1 \%$ chez le rat : La figure 3 montre que l'injection de la carragénine provoque une augmentation progressive du volume de l'œdème chez les rats traités à l'eau distillée $(0,5 \mathrm{ml} / 100 \mathrm{~g})$ durant les six heures de l'expérimentation. L'extrait aqueux des écorces du tronc de $B$. coriacea ne provoque pas une diminution du volume de l'œdème des rats pendant les deux premières heures. application du test t de student, suivi de l'analyse de la variance (ANOVA) et de Tukey's multiple comparaison test. Le seuil de significativité a été fixé à $p<0,05$.

Cependant, de la 3ème à la 6ème heure, cet extrait (200 et $400 \mathrm{mg} / \mathrm{kg}$ ) provoque une diminution du volume de l'œdème, plus importante que celle du diclofénac ( 5 $\mathrm{mg} / \mathrm{kg}$ ). Les pourcentages d'inhibition de l'œdème à $4 \mathrm{~h}$ et $6 \mathrm{~h}$ étant respectivement de 57,05 et $72,56 \%$ pour la dose $200 \mathrm{mg} / \mathrm{kg}, 61,96$ et $69,23 \%$ pour la dose de 400 $\mathrm{mg} / \mathrm{kg}$ et 50,30 et $62,56 \%$ pour le diclofénac à $5 \mathrm{mg} / \mathrm{kg}$ (Figure 4). 


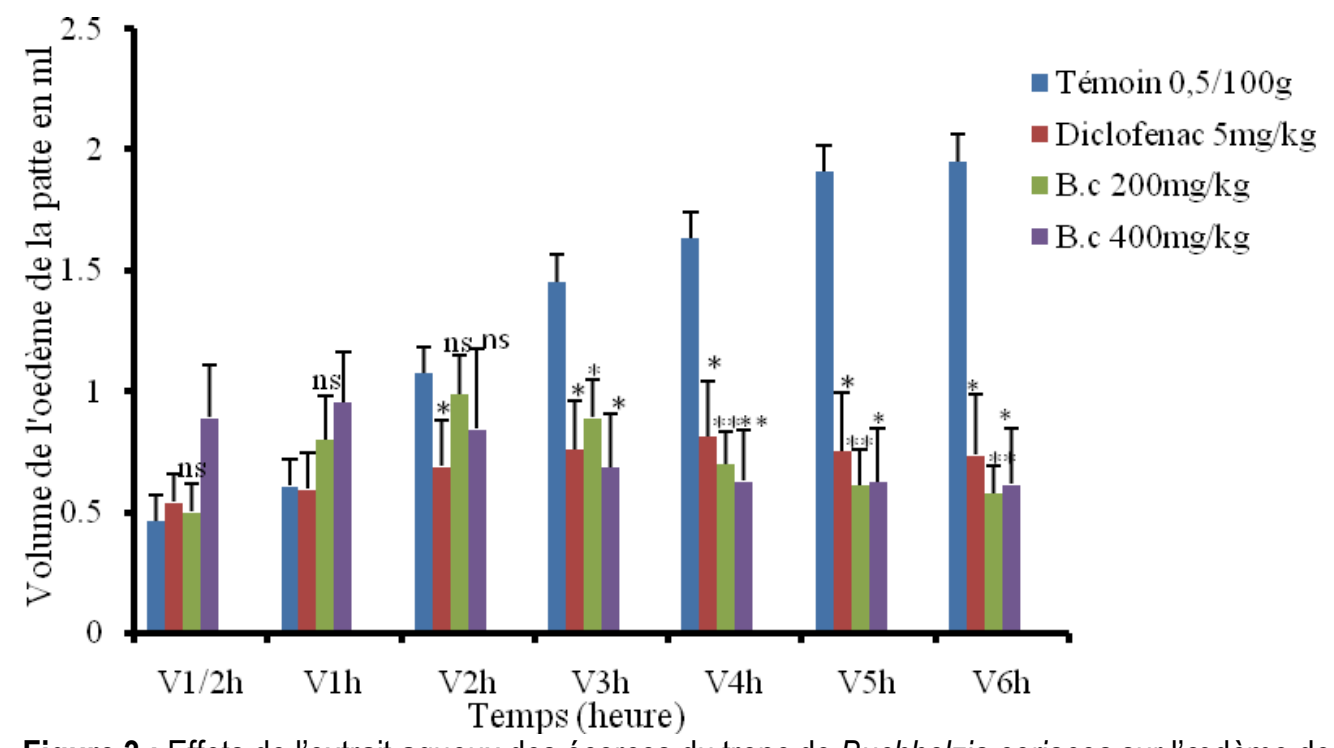

Figure 3 : Effets de l'extrait aqueux des écorces du tronc de Buchholzia coriacea sur l'œdème de la patte induit par la carragénine $1 \%$ chez le rat. ns= non significative, ${ }^{*} P<0,05,{ }^{*} P<0,01$ différences significatives par rapport au lot témoin (eau distillée). Chaque valeur représente la moyenne \pm ESM
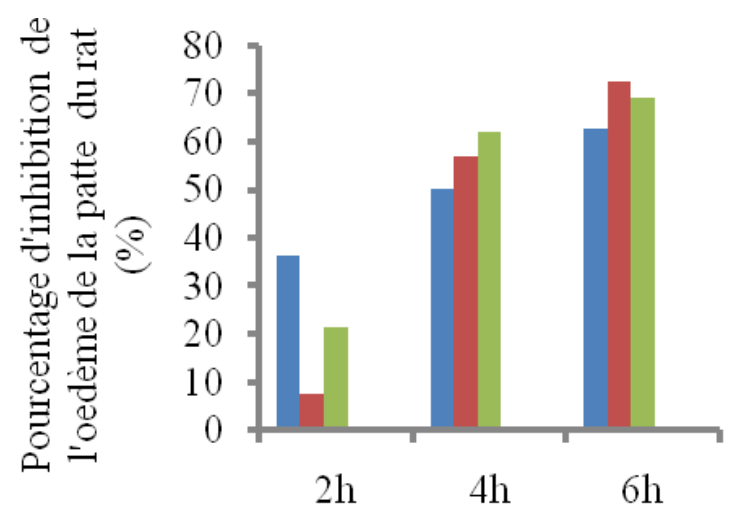

- Diclofenac $5 \mathrm{mg} / \mathrm{kg}$

B.c $200 \mathrm{mg} / \mathrm{kg}$

B.c $400 \mathrm{mg} / \mathrm{kg}$

Temps en heures

Figure 4 : Inhibition de l'inflammation aiguë par le Diclofenac et de l'extrait aqueux des écorces de B. coriacea (B. c)

Effet de l'extrait éthanolique des écorces du tronc de $B$. coriacea sur l'inflammation aigue induite par la carragénine $1 \%$ chez le rat: L'injection de la carragénine sous le coussinet plantaire provoque une augmentation progressive du volume de l'œdème chez les rats traités à la solution de $\mathrm{NaCl} 0,9 \%(1 \mathrm{ml} / 100 \mathrm{~g})$ durant les six heures de l'expérimentation. Chez les rats traités aussi bien avec l'extrait éthanolique des écorces du tronc de B. coriacea (200 et $400 \mathrm{mg} / \mathrm{kg}$ ) qu'avec le Diclofénac $(5 \mathrm{mg} / \mathrm{kg})$, il est observé une importante diminution du volume de l'œdème dès la deuxième heure (figure 5). Les pourcentages respectifs d'inhibition aux temps $2 \mathrm{~h}, 4 \mathrm{~h}$ et $6 \mathrm{~h}$ étant de 65,$80 ; 78,57$ et $87,34 \%$ pour la dose de $200 \mathrm{mg} / \mathrm{kg}, 58,18 ; 92,91$ et $98,19 \%$ pour la dose de $400 \mathrm{mg} / \mathrm{kg}$ de cet extrait et 69,$58 ; 82,57$; et $88,60 \%$ pour la dose de $5 \mathrm{mg} / \mathrm{kg}$ du Diclofénac (figure 6 ). 


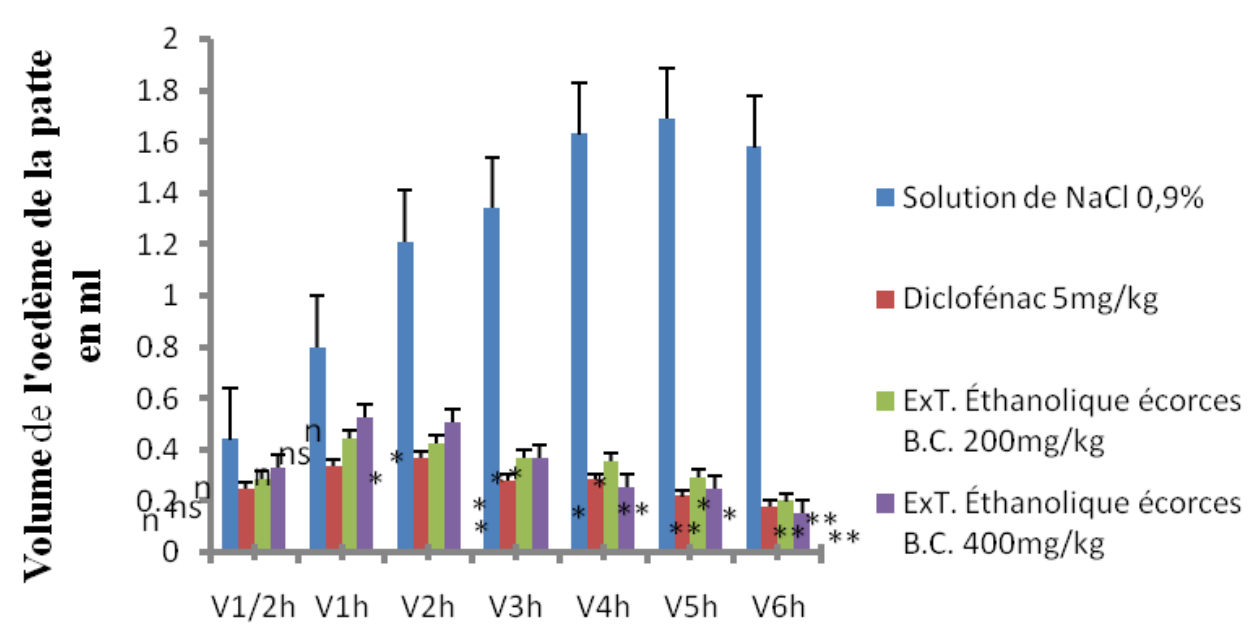

Temps en heure

Figure 5. Effets de l'extrait éthanoïque des écorces de $B$. coriacea sur l'œdème de la patte induit par la carragénine $1 \%$ chez le rat ns $=$ non significative, ${ }^{*} P<0,05,{ }^{* *} P<0,01,{ }^{* * *} p<0,001$ différences significatives par rapport au lot témoin $(\mathrm{NaCl}, 9 \%)$. Chaque valeur représente la moyenne $\pm \mathrm{ESM}$.

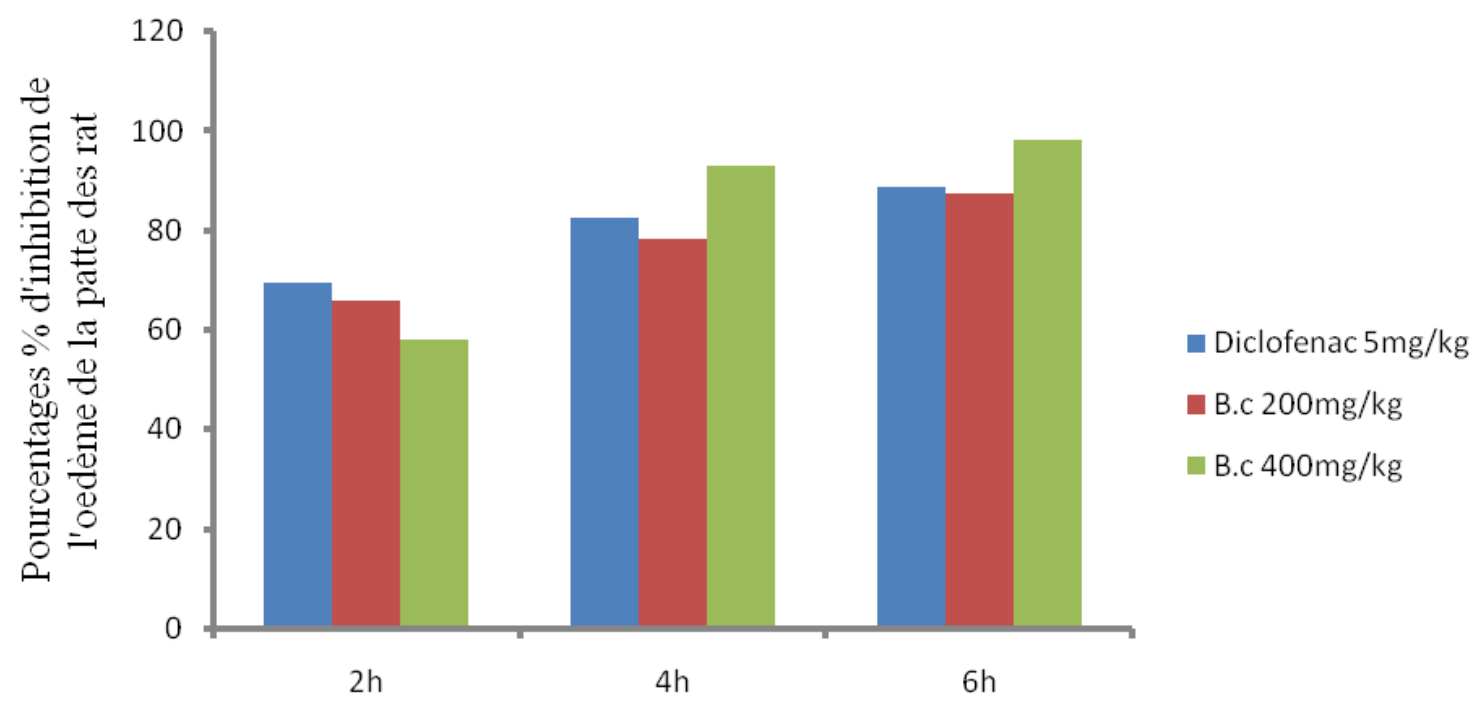

Temps en heures

Figure 6 : Inhibition de l'inflammation aiguë par le diclofenac et de l'extrait éthanolique des écorces de $B$. coriacea (B. c)

Effets de l'extrait éthanolique pâteux des écorces du tronc de $B$. coriacea sur les plaies induites chez les rats wistar: Le tableau 1 montre que le délai de cicatrisation chez les rats témoins ( $\mathrm{NaCl} 0,9 \%$ ) est d'environ 22 jours, alors que ce délai est de 18 jours chez les rats traités avec la pâte de l'extrait éthanolique des écorces du tronc de B. coriacea $(200 \mathrm{mg} / \mathrm{kg})$ et avec la
Bétadine soit une différence du délai de cicatrisation de 4 jours. Les pourcentages de cicatrisation des plaies baissent à $0 \%$ au 22 ème jour chez les rats témoins $(\mathrm{NaCl}$ $0,9 \%$ ) et 18 ème jour chez les rats traités avec la Bétadine ainsi qu'avec cet extrait. Les figures 7, 8, 9, 10, 11 et 12 montrent l'évolution des plaies au $8^{\text {ème }}$ et 14ème jour du traitement. 
Epa et al. J. Appl. Biosci. 2015 Effet anti-inflammatoire et cicatrisant des extraits aqueux et éthanolique des écorces du tronc de Buchholzia coriacea Engl. (Capparidaceae)

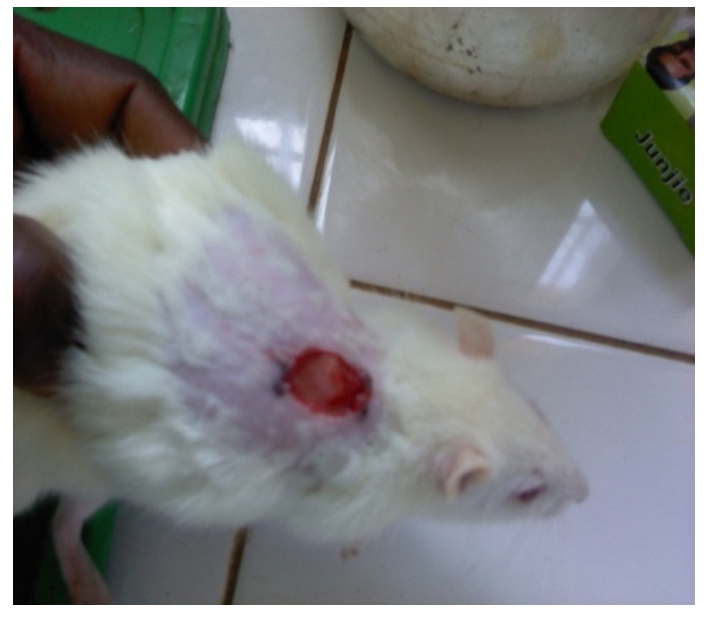

Figure 7 : Rat témoin non traité au 8ème jour d'observation

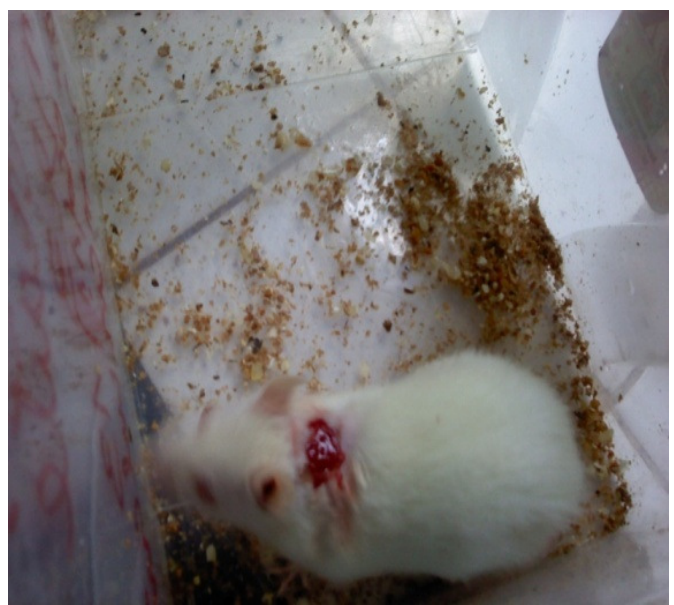

Figure 8: Rat traité avec la Bétadine, au 8ème jour

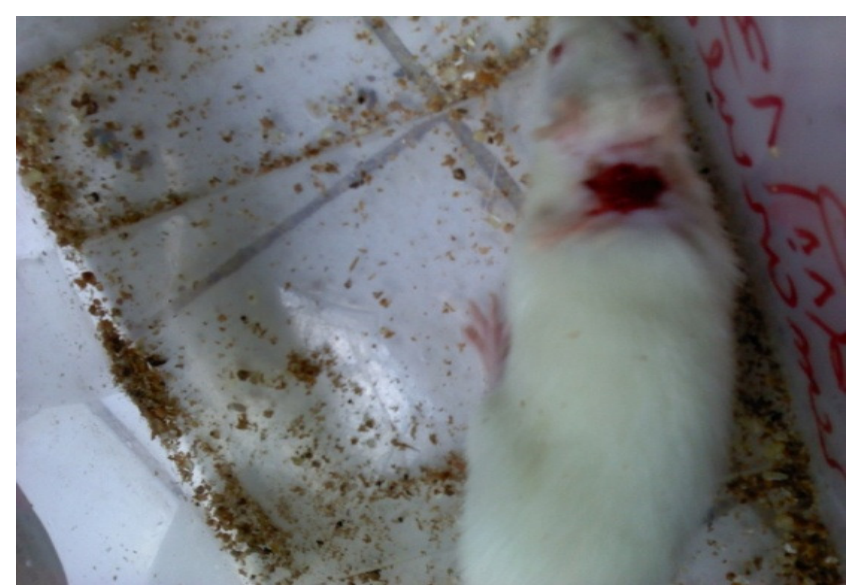

Figure 9 : Rat traité avec l'extrait éthanolique, plaie au 8ème jour

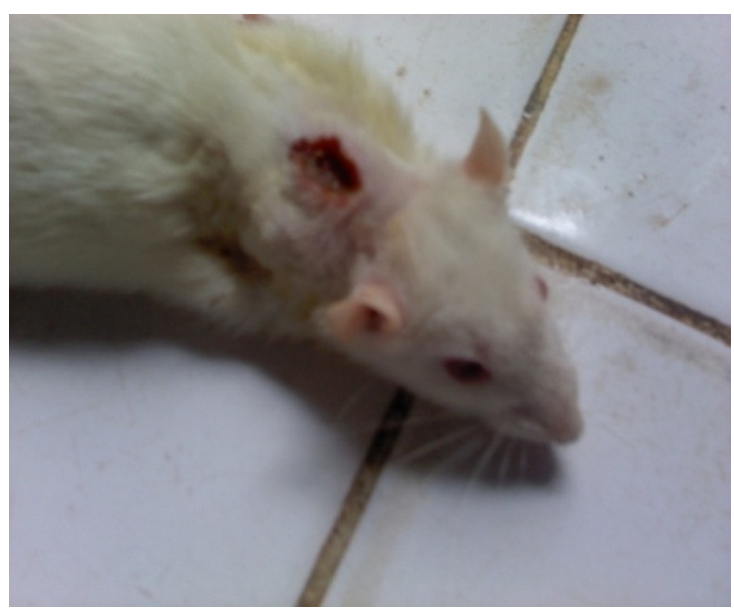

Figure 10: Rat témoin plaie au14ème jour d'observation

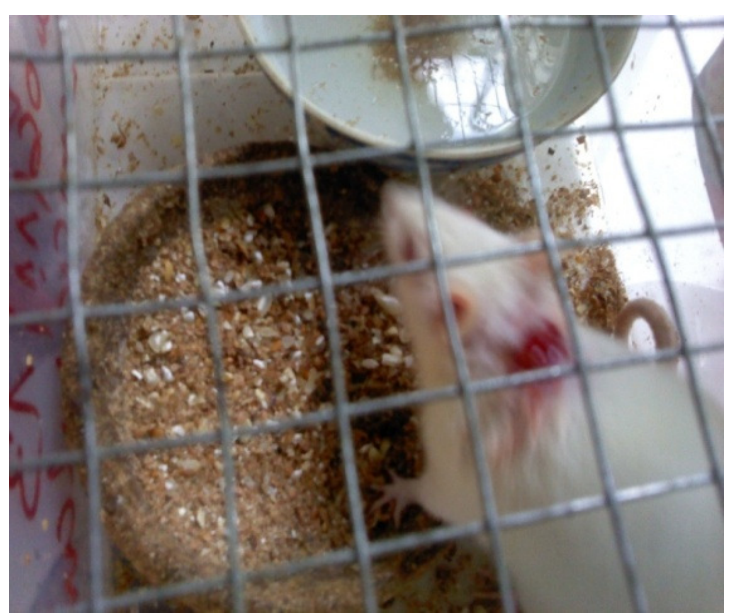

Figure 11 : Rat traité avec la Bétadine, plaie au 14 ème jour 


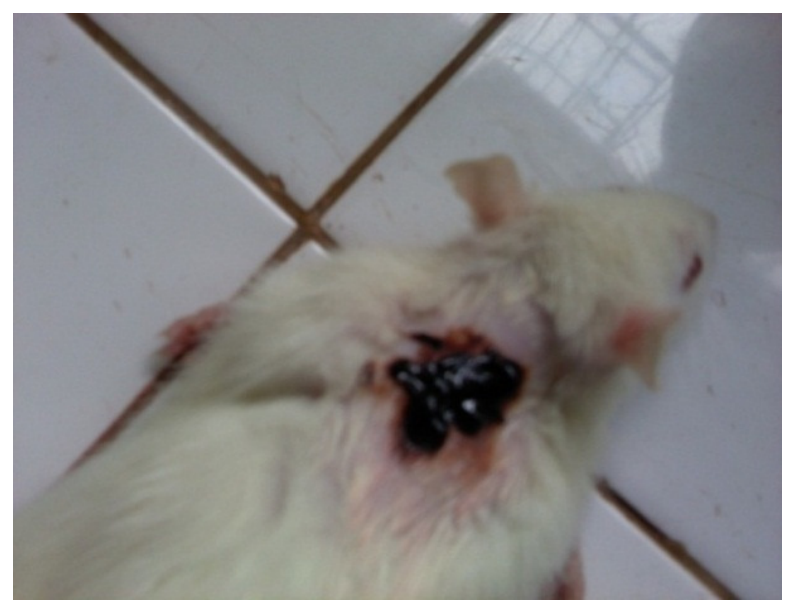

Figure 12 : Rat traité avec l'extrait éthanolique pâteux, plaie au 14ème jour

Tableau 1 : Effets de l'extrait éthanoliquepâteux des écorces de B. coriacea sur le délai de cicatrisation des plaies

\begin{tabular}{|l|l|l|l|}
\hline \multirow{3}{*}{ Jours } & Pourcentage de cicatrisation (\%) & \\
\cline { 2 - 4 } & NaCl 0,9\% (10 ml/kg) & Bétadine (2 ml) & B. coriacea $(\mathbf{2 0 0} \mathbf{~ m g} / \mathbf{k g})$ \\
\hline$J_{1}$ & 100 & 100 & 100 \\
$J_{2}$ & $96 \pm 1,04$ & $84 \pm 2,92$ & $80 \pm 1,58$ \\
$J_{4}$ & $90 \pm 1,58$ & $69 \pm 8,27$ & $69 \pm 1,87$ \\
$J_{6}$ & $80 \pm 274$ & $54 \pm 9,27$ & $60 \pm 2,74$ \\
$J_{8}$ & $66 \pm 2,92$ & $39 \pm 2,92$ & $43 \pm 2,55$ \\
$J_{10}$ & $54 \pm 5,10$ & $23 \pm 3,74^{*}$ & $23 \pm 2,55^{*}$ \\
$J_{12}$ & $42 \pm 3,71$ & $19 \pm 8,28^{* *}$ & $13 \pm 2,00^{* *}$ \\
$J_{14}$ & $31 \pm 4,85$ & $6 \pm 1,00^{* * *}$ & $7 \pm 1,00^{* * *}$ \\
$J_{16}$ & $19 \pm 3,32$ & $1 \pm 1,00^{* * *}$ & $1 \pm 1,00^{* * *}$ \\
$J_{18}$ & $12 \pm 2,1$ & $0 \pm 0,00$ & $0 \pm 0,00$ \\
$J_{20}$ & $2 \pm 0,00$ & $0 \pm 0,00$ & $0 \pm 0,00$ \\
$J_{22}$ & $0 \pm 0,00$ & $0 \pm 0,00$ & $0 \pm 0,00$ \\
\hline
\end{tabular}

Les résultats sont exprimés en moyenne $\pm E S M$ avec $n=5 ; n s=$ non significative ${ }^{\star} p<0,05 ;{ }^{* *} p<0,01 ;{ }^{* * *} p<0,001$ différence significative par rapport aux rats témoins.

\section{DISCUSSION}

Les résultats de la présente étude montrent que les extraits aqueux et éthanolique des écorces du tronc de $B$. coriacea s'opposent à l'augmentation du volume de l'œdème de la patte du rat induit par la carragénine $1 \%$. La carragénine, est un mucopolysaccharide qui induit un maximum d'œdème à partir de la 3ème heure qui suit son injection (Elion Itou et al., 2014). Ces extraits possèdent donc un effet anti-inflammatoire. L'effet anti-inflammatoire de l'extrait aqueux des écorces du tronc de $B$. coriacea (200 et $400 \mathrm{mg} / \mathrm{kg}$ ) est observé à partir de la 3 ème heure alors que celui de l'extrait éthanolique (200 et $400 \mathrm{mg} / \mathrm{kg}$ ) est observé dès la $2^{\mathrm{èm}}$ heure. Les études antérieures ont montré aussi les effets anti-inflammatoires des extraits aqueux et méthanolique des graines et feuilles de $B$. coriacea (Ayoola et al, 2011; Ibrahim et Fagbonun, 2013a et 2013b ; Adu-Amoah et al, 2014 ; Enechi et Nwodo, 2014) et des extraits d'autres plantes (Agbonon, 2000 ; Pham, 2006; Adu-Amoah et al, 2014). La pharmacopée concède à la plupart des composés phénoliques une activité anti-inflammatoire (Chika et al, 2012 ; Duru et al, 2013 ; Ibrahim et Fagbonun, 2013a ; Enrechi et Nwodo, 2014). Pour cela, on pourrait penser que l'activité anti-inflammatoire des écorces du tronc de $B$. coriacea serait due aux flavonoïdes et aux tanins qu'elles contiennent. II a été rapporté que les flavonoïdes, antioxydants naturels, joueraient un rôle très important dans le traitement des inflammations, des tumeurs et des affections bactériennes (Nassis et al, 1992 ; Ezeja et al, 
2011 ; I brahim et Fagbonun, 2013a et 2013b ; Duru et al, 2013). Dans les conditions de notre étude, l'effet antiinflammatoire de l'extrait éthanolique semble être plus efficace que celui de l'extrait aqueux. En effet, le pourcentage d'inhibition du volume de l'œdème avec l'extrait éthanolique (200 mg/kg) est de $87,34 \%$ à la 6 ème heure alors qu'avec l'extrait aqueux à la même heure et avec la même faible dose il est de $72,56 \%$. C'est ainsi que les effets cicatrisants n'ont été recherchés sur les plaies induites chez le rat Wistar qu'avec l'extrait éthanolique pâteux des écorces du tronc de $B$. coriaceaà la dose de $200 \mathrm{mg} / \mathrm{kg}$. Les résultats obtenus montrent que les plaies opératoires des rats normaux traitées tous

\section{BIBLIOGRAPHIE}

Abena A. A., Diatewa M., Gakosso G., Gbeassor M., Hondi-Assah Th., Ouamba J.M., 2003. Analgesic, antipyretic and anti-inflammatory effects of essential oil of Lippia multiflora. Fitoterapia 74 : 231-236.

Adjanohoun E., Ahyi M. R. A., Assi L. A., Baniakina J., Chibon P., Cusset G., Doulou V., Enzanza A., Eymé J., Goudoté E., Keita A., Mbemba C., Mollet J., Moutsamboté J. M., Mpati J., Sita P., 1988. Contribution aux études ethnobotaniques et floristiques en République Populaire du Congo. Agence de Coopération Culturelle et Technique (A.C.C.T.), Paris, 605 P.

Adu-Amoah L., Agyare C., Kisseih E., Ayande P. G., Mensah B. K., 2014. Toxicity assessment of Erythrophleum ivorense and Parquetina nigrescens. Toxicology Reports 1: 411-420.

Agbonon A., 2000. Study of the properties antiinflammatory drugs of the root of Pluchea ovalis sea-green D. C. (Asteraceae) in the rat. Mémoire de DEA de Pharmacologie physiologie, Université de Lomé-Togo, p 25.

Ayoola M. D., Gbolade A. A., Ibitoye S. F., Moody J. O., 2011. In vivo inflammatory methanol extract of Treculla Africana and Buchholzia coriacea Engl. Journal Pharm. Sci. 10:27-31.

Barbara A. Schmidt and Valerie Horsley (2013). Intradermal adipocytes mediate fibroblast recruitment during skin wound healing. Development $140: 1517-1527$.

Bassyouni FA, ASA All, Haggag WM, Mahmoud M, Sarhan MMA, Abdel-Rehim M. (2012). Antimicrobial, anti-inflammatory, and antinociceptive activities of triazole, pyrazole, oxadiazine, oxadiazole, and sugar hydrazone-5nitroindoline-2-one derivatives and a study of les deux jours ont été cicatrisées à partir du $18^{\mathrm{me} e}$ jour, lorsque l'extrait pâteux était appliqué une seule fois le jour du traitement. Les substances naturelles contenues dans cet extrait ne perturberaient pas la séquence normale des événements du processus aboutissant à une bonne cicatrisation. La cicatrisation, comme le rappellent Manjunatha et al (2005) , Falanga et al (2006), Michalik et Wahli (2006), Shuka et al (2007), Korkmaz et al (2008), Li $\mathrm{J}-\mathrm{X}$ et al (2014), Zhang et al (2014), est un phénomène automatique suivant une inflammation de quelque nature que ce soit. Un retard de cicatrisation peut être causé par une infection bactérienne et/ou une immunodépression subséquente.

their computational chemistry (part II). Egyptian Pharmaceutical Journal, 11: 136-143.

Bouquet A., 1969. Féticheurs et médecine traditionnelle du Congo (Brazzaville). Mémoires O.R.S.T.O.M., Paris, $36: 115$ p.

Bouquet A., Jacquot A., 1967. Essai de géographie linguistique sur quelques plantes médicinales du Congo-Brazzaville, 12p.

Bouquet A., 1969. Médecine traditionnelle et pharmacopée: contribution aux études ethnobotaniques et floristiques en république populaire du Congo. Rapport présenté à I'ACCT, Paris, 1988, 605p.

Charlotte D. F., 2012. Cicachar html Histopathologie et mécanisme de la cicatrisation $6: 12-25$.

Chen S. M., Ward S. I, Olutoye O. O, Diegelmann R. F., kelman Cohen I., 1997. Ability of chronic wound fluids to degrade peptide growth factors is associated with increased level of elastase activity and diminished levels of proteinase inhibitors.Wound Repair Regen 5(1):23-32.

Chika E., Moses I., Chigozie U., Olisa A., Ifeanyichukwu I., Michael A., Charles E., (2012). Evaluation of anti-bacterial activity of the leave extracts of Buchholziacoriacea. S. Phar. Biol. Res. 2(4):204-208.

Diafouka A. J. P., 1997. Analyze uses of the medicinal plants in 4 areas of Congo- Brazzaville. Thesis of doctorate, Université Libre de Bruxelles, Faculté des Sciences, Laboratoire de Botanique et systématique, Phytosociology, 431 p.

Duru M., Ugbogu A., Amadi B., Odika P. O., Chima-Ezika O., Anudike J., Osuocha K., 2013. Chemical constituents of Buchholzia coriacea seed. Acadmia.edu 70: 39-41. 


\section{Epa et al. J. Appl. Biosci. 2015 Effet anti-inflammatoire et cicatrisant des extraits aqueux et éthanolique}

des écorces du tronc de Buchholzia coriacea Engl. (Capparidaceae)

Enechi O. C., Nwodo O. F. C., 2014. Anti-ulcer and gastric, anti-secretory activities of seed extract of Buchholzia coriacea in wistar albinos rats. African Journal of Biotechnology 13: 27552761.

Elion Itou R.D.G., Sanogo R., Etou Ossibi A.W., Nsondé Ntandou F.G., Ondelé R., Pénemé B.M., Okiémy Andissa N., Diallo D., Ouamba J.M., Abena A.A., (2014). Anti-inflammatory and analgesic effects of aqueous extract of stem bark of Ceiba pentandra Gaertn. Pharmacology \& Pharmacy, $5: 1113-1118$

Ezeja M. I., Ezeigbo I. I, Madubuike K. G. 2011. Analgesic activity of the methanolic seed extract of Buchholzia coriacea. Research Journal of Pharmaceutical, Biological and Chemical Sciences, 2: 187-193.

Ezekiel O. O. Onyeoziri N. F., 2009. Preliminary studies one the antimicrobial properties of Buchholzia coriacea (wonderful cola). Department of Food Technology, University of Ibadan, Ibadan, Oyo State, Nigeria African Newspaper of Biotechnology 8 (3): 472-474.

Falanga V., Saap L. J., Ozonoff A., 2006. Wound bed score and its correlation with healing of chronic wounds. Dermol ther 19(6):383-390.

Félix-Silva J., Giordani R. B., Da Silva Jr A. A. , Zucolotto S. M., Fernandes-Pedrosa M. F., 2014. Jatropha gossypiifolia L. (Euphorbiaceae): A Review of Traditional uses, phytochemistry, Pharmacology, and Toxicology of this medical Plant. Evid Based Complement Alternat Med., $32 \mathrm{p}$.

Fred-jaiyesimi A, Ogbole O., Anthony O., Egbebumni O., 2011. Larvicidal effect of the fart. Ether, chloroform fractions and methanol extract of Buchholziacoriacea Engle seed. International newspaper of Pharmaceutical sciences research 2(7) : 1736-1739.

Fujino T., Takeuchi A,, Maruko-Ohtake A., Ohtake Y., Satoh J., Kobayashi T., Tanaka T., Ito H., Sakamaki R., Kashimura R., Ando K., Nishimaki-Mogami T., Ohkubo Y., Kitamura N., Sato R., Kikugawa K., Hayakawa M., 2012. Critical role of farnesoid $X$ receptor for hepatocellular carcinoma cell proliferation. $J$ Biochm. 152: 577-586.

Ibrahim T. A., Fagbonun E. D., 2013a. Phytochemical and mineral quality of dried seeds of Buchholzia coriacea. Journal of Applied
Phytotechnology in Environment Sanitation, 2: $121-126$.

Ibrahim T. A. and Fagbonun E. D. 2013b. Antibacterial and Antifungal Activity of Ethanolic and Methanolic Extract of Dried Seeds of Buchhlozia coriacea. Greener Journal of Agricultural Sciences 3:458-463.

Korkmaz B., Moreau T., Gauthier F., 2008. Neutrophil elastase, proteinase 3 and cathepsin $G$ : physicochemical properties, activity and physiopathological functions. Biochimie 90:22742.

Lawal I. O., Uzokwe, Igboango A. B. I., Awosan A. F., Nwogwugwu O. J., Faleye B., Olatunji B. F., Adesoga A. A., 2010. Ethno medicinal information one collation and identification of some medicinal seedlings in research institute of south.West Nigeria. African newspaper Pharmacy and Pharmacology 4(1) : 001-007.

Li J-X, Thorn D. A., Qiu Y., Peng B. W., Zhang Y., 2014. Antihyperalgesic effects of imidazoline $I_{2}$ receptor ligands in rat models of inflammatory and neuropathic pain. $\mathrm{Br} J$ Pharmacol 171(6):1580-1590.

Manjunatha B. K., Vidya S. M., Rashmi K. V., Mankani K. L., Shilpa H. J., Singh S., Jagadeesh D., 2005. Evaluation of wound-healing potency of Vernoniaarborea Hk. Indian journal of pharmacology, 37:223-226.

Michalik L., and Wahli W. (2006). Involvement of par nuclear receptors in tissue injury and wound repair.JClin Invest. 116(3): 598-606.

Narayanan N., Thirugnana S. P., Viswanathan S., Kamappa Reddy M., Vijayasekaran V., Sukumar E., 2000. Antipyretic, antinociceptive and anti- inflammatory activity of Premna herbacea roots. Fitoterapia 71 : 147-153.

Nassis C. Z., Haebisch E. M., Giesbrecht A. M., 1992. Antihistamine activity of Bryophyllum calycinum. Brazilian journal of medicine and Biological Research 25 : 929-936.

Nwachukwu . I, Duru M. K. C., Amadi B. A., Nwachukwu I. O., 2014. Comparative Evaluation of Phytoconstituents, Antibacterial Activities and Proximate Contents of Fresh, Oven Dried Uncooked and Cooked Samples of Buchholzia coriacea Seed and Their Effects on Hepatocellular Integrity. International Journal of Pharmaceutical Science Invention $3: 41-49$. 
OMS, 2013. Stratégie de l'OMS pour la médecine traditionnelle pour 2014-2023. Genève (Suisse), $75 \mathrm{p}$.

Pham C. T., 2006. Neutrophil serine proteases : specific regulators of inflammation. Nat Rev Immuno l6: 541-50.

Sagliyan A., Ceribasi A. O., Gunay C., Han M. C., Benzer F., Kandemir, 2010. Effects of dietary supplementation with whey proteins on surgical wound healing in rats. Revue Méd.161(10) :455462.

Shuka V. K., Shuka D., Tiwary S. K., Agrawal S., Rastogi A., 2007. Evaluation of $\mathrm{pH}$ measurement as a method of wound assessement. J wound care 16 (7) : 291-94.
Viel E., Ben Ammar M. S., Eledjam J. J., 2006. AINS, inhibiteurs de la COX-2. In: Evaluation et traitement de la douleur. Amsterdam, Elsevier, $603-616$

WHO, 2000. Promoting the Role of Traditional Medicine in Health Systems: a Strategy for the African Region 2001-2010, document AFR/RC50 /Doc.9/R). Harare, $15 \mathrm{p}$.

Zhang W., Yu J., Dong Q., Zhoo H., Li F., Li H. 2014. A mutually beneficial relationship between hepatocytes and Cardiomyocytes mitigates. Doxorubicin-induced toxicity. Toxicology Letters 227: 157-163. 